\title{
Characterization of the Opportunistic Service Time in Cognitive Radio Networks
}

\author{
Miguel Luís, Rodolfo Oliveira, Senior Member, IEEE, Rui Dinis, Senior Member, IEEE, \\ and Luis Bernardo, Member, IEEE
}

\begin{abstract}
This paper studies the service time required to transmit a packet in an opportunistic spectrum access scenario, where an unlicensed secondary user (SU) transmits a packet using the radio spectrum licensed to a primary user (PU). Considering a cognitive radio network, it is assumed that during the transmission period of an SU multiple interruptions from PUs may occur, increasing the time needed to transmit a packet. Assuming that the SU's packet length follows a geometric distribution, we start by deriving the probability of an SU transmitting its packet when $k>0$ periods of PU's inactivity are observed. As the main contribution of this paper, we derive the characteristic function of the service time, which is further used to approximate its distribution in a real-time estimation process. The proposed methodology is independent of the SUs' traffic condition, i.e., both saturated or non-saturated SU's traffic regime is assumed. Our analysis provides a lower bound for the service time of the SUs, which is useful to determine the maximum throughput achievable by the secondary network. Simulation results are used to validate the analysis, which confirm the accuracy of the proposed methodology.
\end{abstract}

Index Terms-Service time, cognitive radio networks, queueing analysis, performance evaluation and modeling.

\section{INTRODUCTION}

$\mathbf{C}$ OGNITIVE radio networks (CRNs) are an effective solution to alleviate the increasing demand for radio spectrum [1], [2]. In CRNs, the transmission channel is licensed to the primary users (PUs) while secondary users (SUs) opportunistically access the licensed channel when it is not being used by any PU. Accordingly, a SU needs to detect a vacant channel for transmission, and vacates it when a PU starts to use the channel. A SU should operate transparently to the PUs and avoid causing interference to PUs acting as receivers. This fact implies that a SU transmission must be interrupted whenever a PU starts transmitting [3].

Manuscript received July 13, 2015; revised December 29, 2015, June 3, 2016, and August 2, 2016; accepted August 18, 2016. Date of publication August 29, 2016; date of current version October 17, 2016. This work was supported by the Foundation for Science and Technology of the Portuguese Ministry of Education and Science under Project IT (UID/EEA/50008/2013). The associate editor coordinating the review of this paper and approving it for publication was M. Krunz.

M. Luís is with the Instituto de Telecomunicações, 1049-001 Lisbon, Portugal (e-mail: nmal@campus.fct.unl.pt).

R. Oliveira, R. Dinis, and L. Bernardo are with the Instituto de Telecomunicações, 1049-001 Lisbon, Portugal, and also with the Centre of Technology and Systems, UNINOVA - Instituto de Desenvolvimento de Novas Tecnologias, Departamento de Engenharia Electrotécnica, Faculdade de Ciências e Tecnologia, Universidade Nova de Lisboa, 2829-516 Caparica, Portugal (e-mail: rado@fct.unl.pt; rdinis@fct.unl.pt; lflb@fct.unl.pt).

Digital Object Identifier 10.1109/TCCN.2016.2603994
Consequently, the amount of time that a SU needs to transmit a packet depends on the number and duration of the PUs' transmissions. This work focuses on the interval of time from the instant when a packet arrives at the head of the SU transmitting queue, until the instant when its transmission ends, which is denoted hereafter as packet service time or simply service time.

The service interruption of SUs due to PUs may significantly degrade its performance. Multiple mechanisms have already been proposed in the literature to reduce its impact. Among the most relevant ones we can include spectrum handoff [4]-[7], i.e., the possibility of switching the interrupted service to an idle channel, buffering [8], to account for the duration of the interruptions and avoid packet loss, or both spectrum handoff and buffering [9], [10]. Depending on the number of data channels available for transmission, a SU may handoff to a different wireless transmission channel after a PU interruption [11], [12], or remain in the same channel and wait for the PU to vacate the channel [8].

\section{A. Related Literature}

A few works have already characterized the service time considering that no spectrum handoff occurs during the transmission of a SU's packet. Specifically, the average packet service time is studied in [13] and [14] under different preemptive priority queueing models such as $\mathrm{M} / \mathrm{D} / 1$ and $\mathrm{M} / \mathrm{G} / 1$ [15]. Nevertheless, neither of these works examine the role of the size of the SU's and PU's packets, nor the impact of different PU's activity rates on the average service time. Tran et al. [16] consider a preemptive $\mathrm{M} / \mathrm{G} / 1$ priority queueing system to evaluate the average service time when the spectrum is sensed in a discrete and in a continuous way, showing that the average service time of the packets transmitted by a SU not only depends on the arrival rate and size of SU's packets but also on the arrival rate and size of the PU's packets. Reference [17] considers a priority virtual queue to model the joint coexistence of PU and SU traffic. Based on the model, the authors obtain an expression for the expected packet service time of SU's traffic through a M/G/1 preemptive priority queueing model. In [18] the service response time, defined as the duration from the instant that a SU requests a data file from the central controller until it completes the reception, is analyzed for a single channel CRN with centralized control. Reference [18] is extended in [19], where different mathematical expressions for the average service response time of 
elastic data (i.e., variable file-length). By assuming that the PU activity follows an ON-OFF behavior, [18], [19] demonstrate that the preemption reduces the mean response time when the service time requirement follows a heavy tailed distribution.

Reference [20] and [21] assume both scenarios where the SUs may handoff to different wireless channels or may operate in a single channel without handoff. Li and Han [20] propose a simplified model of primary user interruptions based on a Markov chain analysis, being the queueing analysis carried out for the cases of two-server-single-queue (two channels and one SU) and two-queues-single-server (two SUs and one channel). In the former scenario a semi-analytic result is obtained for the generating function of the queue length, while in the latter case the authors derive an expression for the average queue length. The work in [21] characterizes the average service time and average waiting time of a SU's transmission assuming a general primary traffic model and considering three scenarios: i) single-channel, ii) multi-channel considering that once a PU becomes active the SU switches sequentially to the next channel and keeps switching until it finds an unused channel, and iii) multi-channel considering that when a PU becomes active, a SU using the same channel switches and stays on the next channel and transmits if it is free or waits until it becomes free. Numerical results show that the single channel access scheme presents the largest average service time.

More recently, [22] derived expressions for the distribution of the packet service time considering fixed-length SU packets, and continuous and periodic sensing strategies. No spectrum handoff is assumed. As far as we know [22] is the only work where an expression for the distribution of the service time is derived. However, its contribution is only valid for fixedlength SU's data packets. Moreover, the authors only consider the case when SUs are traffic-saturated, and consequently only consider the case when the transmission of the SU starts at the beginning of the PU's inactive period.

\section{B. Novel Contributions}

Mainly focused on practical applications adopting nonpreemptive scheduling techniques, in this work we propose an innovative packet service time analysis of opportunistic access in a CRN. The analysis can be used in both single or multi-channel protocols that consider an exclusive use of the channel by a SU to transmit its packet, and no spectrum handoff is performed during the packet transmission.

Departing from the work in [23], which confirms that the PU activity may be represented by a geometric distribution when small periods of PU's inactivity are observed, we describe several steps required to derive the expressions for the distribution of the SU's service time considering PU interruptions. We start by deriving an expression for the probability of a SU transmitting its packet when $k>0$ periods of PU's inactivity are observed. Then, we extend the previous analysis and derive the characteristic function of the service time, which in turn is used to approximate the distribution of the service time that supports a real-time service time estimation process.
In a nutshell, the main contributions of our work can be summarized as follows:

- We derive the service time characteristic function, which is valid for a general scenario. Contrarily to [22], we consider that: a SU may start its transmission at any instant of the PU's inactive period; the packet length of the SU is variable; both SU's saturated and non-saturated traffic conditions;

- An approximation for the distribution of the service time is proposed, which uses the characteristic function to estimate the parameters of the distribution through the method of moments;

- We provide numerical results obtained with the proposed model, which are compared with simulation results. The accuracy of the proposed methodology is evaluated and different results are presented for: the average service time; the distribution of the service time; the average packet waiting time in the transmission queue; the average number of packets waiting in the transmission queue;

- We assess the accuracy of the average service time obtained in a real-time estimation process, evaluating the accuracy of the estimation for different lengths of the estimation sampling set.

When compared to the works already cited before, our approach also provides high-order statistics of the service time due to the Characteristic Function modeling. This is particularly advantageous in multiple network modeling scenarios where queueing theory tools must be used. Moreover, since the throughput is represented by the inverse of the service time, the proposed analysis provides an upper bound of the throughput when a single SU transmits, which represents the maximum throughput achievable by the Secondary network. This is an important contribution because we may compare this bound with the throughput obtained with practical cognitive Medium Access Control (MAC) schemes to quantify their efficiency in a relative way (quantifying how far the MAC's throughput is from the upper bound proposed in our work). Additionally, as far as we know, this is the first work presenting a generic model-based solution to estimate the service time. The estimation of the service time (as proposed in the paper) can be used in different channels to evaluate their relative occupation by the PUs, which may also be used by the MAC protocols to optimize the channel assignment process or simply rank their availability. But the comparative advantages of our work are not limited to the aforementioned arguments. Because our analysis relies on a discrete time unit and the duration of the packets transmitted by the SUs are multiples of that time unit, our approach is general enough to analyze different practical scenarios, including:

- the case where the frames (at layer 2) of the SUs are transmitted in a single PU OFF period. In this case the discrete time unit adopted in the model must be equal or greater than the duration of the longest SU frame;

- the case where a variable-length file is transmitted by a SU, and the transmission may be spanned by multiple PU OFF periods. In this case the discrete time unit adopted in the model must represent the duration of a SU's frame, 
while the model's packet duration represents the duration of the file.

Consequently, the generic nature of the proposed model may be explored to characterize different scenarios, which brings additional advantages when compared to the related literature.

The rest of the paper is organized as follows. The system's overview is presented in Section II. The characteristic function of the service time is derived in Section III. Section IV proposes an expression for the probability mass function of the service time. In Section V we assess the accuracy of the proposed methodology by comparing the numerical results obtained with the model with several results obtained by simulation. Finally, conclusions are drawn in Section VI.

\section{SYSTEM OVERVIEW}

This work considers the case where one pair of licensed PUs (sender and receiver) is operating in a wireless channel and one pair of SUs (sender and receiver) may use the same channel in an opportunistic way.

The PU transmitter has an ON-OFF behavior, meaning that it is active (i.e., transmitting) during $\mu_{B}$ time units in average, and inactive (not using the channel) during $\mu_{I}$ time units. Hereafter we adopt the terminology active/ON and inactive/OFF PU's periods to denote the amount of time that a PU is $\mathrm{ON}$ or OFF, respectively. The PU's activity is modeled by two random processes. The first one models the duration of the active period, represented by the random variable (r.v.) $B$, and the second one models the duration of the inactive period, represented by the r.v. I. Following the results in [23] obtained with real traces of cellular users, both durations are sampled from geometric distributions with parameters $p_{B}=1 / \mu_{B}$ and $p_{I}=1 / \mu_{I}$, respectively. The probabilities of a PU staying $\mathrm{OFF}$ and $\mathrm{ON}$ are respectively given by $P_{P U}^{I}=\mu_{I} /\left(\mu_{I}+\mu_{B}\right)$ and $P_{P U}^{B}=\mu_{B} /\left(\mu_{I}+\mu_{B}\right)$.

As stated before, the SU transmitter may use the channel whenever it is not being used by the licensed users. For this purpose we assume that the SUs are able to identify the access opportunities through spectrum sensing [24]-[27]. Perfect spectrum sensing is assumed in this work, i.e., we assume that the probabilities of PUs' detection and false alarm are 1 and 0 , respectively.

For time accounting purposes, we consider a constant time duration denoted as a time unit, and the discrete index $k=x$ is used to indicate the $x$-th time unit. A SU's packet of length $\mu_{L}$ requires one or more $\mu_{L}$ time units of PU's inactivity in order to be completely transmitted. However, the proposed analysis may be easily extended for different transmission rates, or even for scenarios where a fixed amount of the discrete time unit is used by the SUs for spectrum sensing. When a SU is able to transmit a packet using only one PU OFF period, the packet service time is exactly $\mu_{L}$ time units. For example, following the hypothetical sequence of PU ON and OFF periods illustrated in Figure 1, this occurs when a SU starts the transmission of a packet of length 2 in the first time unit of the first PU OFF period. However, when the length of the SU's packet is higher than the PU OFF period, a SU will have to interrupt the packet's transmission, and resumes it in the following PU

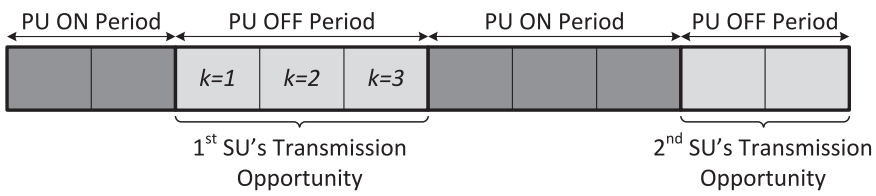

Fig. 1. Hypothetical sequence of PU OFF and ON periods.

OFF period. This case may occur when a packet of length of 4 starts to be transmitted in the second time unit $(k=2)$ of the first PU OFF period illustrated in Figure 1. In this case the packet service time will not only depend on the size of the packet's length, but also on the length of the PU ON and OFF periods.

We highlight that the parametrization of the packet length may represent different practical scenarios. If we consider that the time unit represents the duration of the SU's frame, and if the model's packet length $\mu_{L}$ is exactly one time unit (deterministically), the model's packet transmission only takes one PU OFF period. This case represents a traditional scenario of the SU's service time associated to the SU's frame transmission at layer 2. However, if the model's packet length $\mu_{L}$ is greater then one time unit, the transmission of the model's packet may be spanned by multiple PU OFF periods. This case may represent the SU's service time to transmit a file, as described in Section I.

Since a SU must know the availability of the channel before starting a transmission, we assume that the spectrum sensing information is available in the beginning of the time unit $k$ illustrated in Figure 1. By considering a discrete time analysis, our approach assumes periodic sensing condition: a spectrum sensing procedure takes place on each time unit. In the analysis it is assumed that the SU's time frame lasts at least a time unit, meaning that we neglect the sensing duration. However, the proposed analysis is also applicable for a non-null spectrum sensing duration, by subtracting the sensing time from the time unit. We highlight that the proposed model is generic enough to accommodate different practical scenarios depending on the parameterization of the time unit.

\section{Service Time Characteristic Function}

This section characterizes the service time of a packet transmitted by a SU. The proposed analysis starts by characterizing the number of PU OFF periods that a SU needs to transmit a packet, taking into account the length of the packet as well as the duration of the ON and OFF PU's periods. Then, we derive an expression for the characteristic function (CF) of the packet service time, as well as the first- and the second-order moments, which will be used to approximate the distribution of the packet service time.

\section{A. Service Interruptions}

Let us start by deriving the number of PU OFF periods that a SU needs to transmit a packet, which is represented by the r.v. $I_{\text {periods }}$. The data packet duration generated by the $\mathrm{SU}$ is represented by the r.v. $L$ and, similarly to the PU OFF and ON 
period durations, we consider that it follows a geometric distribution with parameter $p_{L}=1 / \mu_{L}$, meaning that the average packet duration is $\mu_{L}$ time units.

Assuming that a SU starts its transmission in the first time unit of the PU OFF period ( $k=1$ in Figure 1$)$, the SU will be able to transmit the entire data packet in a single PU OFF period if the duration of the period $(I)$ is longer or equal to the duration of the data packet, i.e.,

$$
\operatorname{Pr}\left\{I_{\text {periods }}=1\right\}=\operatorname{Pr}\{I \geq L\} .
$$

Since $I$ and $L$ are independent r.v., the probability of $I-L$ can be found using the cross-correlation between the two r.v. as follows

$$
\operatorname{Pr}\{I-L=t\}=\sum_{k=1}^{+\infty} \operatorname{Pr}\{L=k\} \cdot \operatorname{Pr}\{I=k+t\},
$$

and therefore (1) becomes

$$
\operatorname{Pr}\left\{I_{\text {periods }}=1\right\}=\sum_{k=0}^{+\infty} \operatorname{Pr}\{I-L=k\} .
$$

When a single PU OFF period is not enough to transmit the SU's data packet, the transmission of the packet is resumed to the next PU OFF period. Following the same rationale used in (1), we can write the probability of the SU's transmission be spanned in less or equal than two PU OFF periods as follows

$$
\operatorname{Pr}\left\{I_{\text {periods }} \leq 2\right\}=\operatorname{Pr}\{I+I \geq L\} .
$$

Since the multiple realizations of the duration of a PU OFF period are i.i.d., the probability of $I+I$, the sum of two geometric distributions with the same parameter $p_{I}=1 / \mu_{I}$, is represented by a negative binomial distribution with the parameters $r=2$ and $p=p_{I}$, i.e.,

$$
\operatorname{Pr}\{I+I=t\}= \begin{cases}\left(\begin{array}{c}
t-1 \\
1
\end{array}\right) p_{I}^{2}\left(1-p_{I}\right)^{t-2}, & t \geq 2 \\
0, & t<2 .\end{cases}
$$

In general, we can represent the duration of the sum of $r$ PU OFF periods by the r.v. $I_{r}^{\text {Sum }}$, whose probability mass function $(\mathrm{PMF})$ is given by

$$
\operatorname{Pr}\left\{I_{r}^{\text {Sum }}=t\right\}= \begin{cases}\left(\begin{array}{l}
t-1 \\
r-1
\end{array}\right) p_{I}^{r}\left(1-p_{I}\right)^{t-r}, & t \geq r \\
0, & t<r .\end{cases}
$$

Using (5) in (4), the probability of a SU being able to transmit a data packet lasting $L$ time units using two or less PU OFF periods is given by

$$
\operatorname{Pr}\left\{I_{\text {periods }} \leq 2\right\}=\sum_{k=0}^{+\infty} \operatorname{Pr}\left\{I_{2}^{\text {Sum }}-L=k\right\},
$$

where $\operatorname{Pr}\left\{I_{2}^{\text {Sum }}-L=t\right\}$ follows the same rationale as in (2) and is given by

$$
\operatorname{Pr}\left\{I_{r}^{S u m}-L=t\right\}=\sum_{k=1}^{+\infty} \operatorname{Pr}\{L=k\} \cdot \operatorname{Pr}\left\{I_{r}^{\text {Sum }}=k+t\right\},
$$

for $r=2$.

However, the probability $\operatorname{Pr}\left\{I_{\text {periods }} \leq 2\right\}$ accounts for all the cases when a data packet with length $L$ fits in two or less PU OFF periods, i.e., it also includes the case when the packet is entirely transmitted in a single PU OFF period. Therefore, since we focus on the probability that a data packet is transmitted in exactly $r$ PU OFF periods, we must consider the cases when the packet was not completely transmitted in the previous $r-1 \mathrm{PU}$ OFF periods. Consequently, the PMF of $I_{\text {periods }}$ becomes

$$
\begin{aligned}
\operatorname{Pr}\left\{I_{\text {periods }}=r\right\}= & \sum_{t=0}^{+\infty} \operatorname{Pr}\left\{I_{r}^{\text {Sum }}-L=t\right\} \\
& -\sum_{t=0}^{+\infty} \operatorname{Pr}\left\{I_{r-1}^{\text {Sum }}-L=t\right\},
\end{aligned}
$$

which depends only on the duration of the PU ON period $\mu_{B}$ and the SU's packet length $\mu_{L}$. The term $\sum_{t=0}^{+\infty} \operatorname{Pr}\left\{I_{r}^{\text {Sum }}-\right.$ $L=t$ \} represents the probability of the transmission occupying at most $r$ PU OFF periods, while the term $\sum_{t=0}^{+\infty} \operatorname{Pr}\left\{I_{r-1}^{S u m}-\right.$ $L=t\}$ represents the probability of the transmission occupying at most $r-1$ PU OFF periods.

As stated before, we are assuming that a SU starts the packet transmission in the first time unit of the PU OFF period. In fact, a SU may start the packet transmission in any time unit of the PU OFF period. If we consider a saturated network, where SUs always have a packet to transmit, the beginning of a new packet transmission will only depend on when the previous transmission was finished. On the other hand, if we consider a non-saturated network, the beginning of a new packet transmission also depends on the arrival instant of a new data packet. However, since we are considering geometric distributions to represent the duration of the PU ON and OFF periods, the memoryless property of these distributions can be used to justify that the number of PU OFF periods that a SU needs to transmit a packet does not depend on which time unit the packet transmission has started.

\section{B. Characteristic Function}

The definition of the characteristic function $(\mathrm{CF})$ of the service time is based on the number of PU's OFF periods observed during the SU's transmission. The service time of a SU's data packet is defined as the interval from the instant when a packet arrives the head of the transmitter's queue, until the instant when its transmission ends. The packet service time, measured in discrete units of time, is represented by the r.v. $S$, while its $\mathrm{CF}$ is denoted by $\Phi_{S}(\omega)$, with $\omega \in \mathbb{R}$.

To derive the $\mathrm{CF}$ in a comprehensive manner, we divide its derivation in three different scenarios represented by the following Lemmas. We start by deriving the $\mathrm{CF}$ of the packet service time for a specific scenario (Lemma 1) and then we improve to a more generic scenario (Lemma 3).

Lemma 1: When a SU always has a packet to transmit and the previous packet transmission ends at any time unit of the PU OFF period except the last one, e.g., $k=1$ and $k=2$ of the first PU OFF period illustrated in Figure 1, the CF of the packet service time is given by

$$
\begin{aligned}
\Phi_{S}(\omega)= & \sum_{r=1}^{+\infty} \operatorname{Pr}\left\{I_{\text {periods }}=r\right\} \\
& \times \frac{p_{L} e^{j \omega}}{1-\left(1-p_{L}\right) e^{j \omega}}\left(\frac{p_{B} e^{j \omega}}{1-\left(1-p_{B}\right) e^{j \omega}}\right)^{r-1} .
\end{aligned}
$$


Proof: When a SU is able to transmit the entire packet in a single PU OFF period the service time depends only on the size of the data packet, i.e.,

$$
\Phi_{S}(\omega)=\Phi_{L}(\omega),
$$

where $\Phi_{L}(\omega)$ represents the CF of the packet duration in terms of $k$ time units and is given by

$$
\Phi_{L}(\omega)=\frac{p_{L} e^{j \omega}}{1-\left(1-p_{L}\right) e^{j \omega}},
$$

which represents the $\mathrm{CF}$ of a geometric distribution for $k>0$ ( $j$ represents the imaginary unit) [28]. If a SU requires two PU OFF periods to transmit the packet, the packet service time includes one PU ON period as follows

$$
\Phi_{S}(\omega)=\Phi_{L}(\omega) \cdot \Phi_{B}(\omega)
$$

where $\Phi_{B}(\omega)$ represents the $\mathrm{CF}$ of the duration of a PU ON period, and is given by

$$
\Phi_{B}(\omega)=\frac{p_{B} e^{j \omega}}{1-\left(1-p_{B}\right) e^{j \omega}} .
$$

Following the same rationale, under the assumptions considered in this Lemma, we can write the CF of the service time based on the number of required PU OFF periods as follows

$$
\Phi_{S}(\omega)=\sum_{r=1}^{+\infty} \operatorname{Pr}\left\{I_{\text {periods }}=r\right\} \cdot \Phi_{L}(\omega) \cdot \Phi_{B}^{r-1}(\omega) .
$$

Lemma 2: When a SU always has a packet to transmit and the previous packet transmission ends at any time unit of the PU OFF period, e.g., $k=1, \ldots, 3$ of the first PU OFF period in Figure 1, the CF of the service time is given by

$$
\begin{aligned}
\Phi_{S}(\omega)= & \left(1-p_{I}\right) \sum_{r=1}^{+\infty} \operatorname{Pr}\left\{I_{\text {periods }}=r\right\} \\
& \times \frac{p_{L} e^{j \omega}}{1-\left(1-p_{L}\right) e^{j \omega}}\left(\frac{p_{B} e^{j \omega}}{1-\left(1-p_{B}\right) e^{j \omega}}\right)^{r-1} \\
& +p_{I} \sum_{r=1}^{+\infty} \operatorname{Pr}\left\{I_{\text {periods }}=r\right\} \\
& \times \frac{p_{L} e^{j \omega}}{1-\left(1-p_{L}\right) e^{j \omega}}\left(\frac{p_{B} e^{j \omega}}{1-\left(1-p_{B}\right) e^{j \omega}}\right)^{r} .
\end{aligned}
$$

Proof: Considering the definition of service time described before, if the previous transmission ends at the last time unit of the PU OFF period $(k=3$ of the first PU OFF period in Figure 1) a new packet arrives at the head of the SU's buffer queue in the first time unit of a PU ON period. When this occurs, the packet service time includes an entire PU ON period before any PU OFF period. Therefore, if a SU is able to transmit the entire packet in a single PU OFF period, the service time is given by

$$
\Phi_{S}(\omega)=\Phi_{L}(\omega) \cdot \Phi_{B}(\omega) .
$$

Following the same rationale as before, if two PU OFF periods are required to transmit the entire packet, the service time of a data packet that arrived at the head of the queue in the first time unit of a PU ON period is given by

$$
\Phi_{S}(\omega)=\Phi_{L}(\omega) \cdot \Phi_{B}^{2}(\omega) .
$$

In general, the service time of a data packet arriving at the head of the SU's buffer in the first time unit of the PU ON period is given by

$$
\Phi_{S}(\omega)=\sum_{r=1}^{+\infty} \operatorname{Pr}\left\{I_{\text {periods }}=r\right\} \cdot \Phi_{L}(\omega) \cdot \Phi_{B}^{r}(\omega) .
$$

Let $\gamma_{I}$ be the probability of a packet arriving at the head of the SU's buffer queue during a PU OFF period. Considering that Lemma 2 assumes that the previous transmission ends at any slot of the PU OFF period and both PU ON and OFF periods follow geometric distributions, the memoryless property of the geometric distribution indicates that the probability of the previous SU's transmission ending in a given slot of the PU OFF period is equal for any slot $1, \ldots, \mu_{I}$. However, because this lemma also considers that a $\mathrm{SU}$ always has a packet to transmit, only the cases when the previous transmission ends at slots $1,2, \ldots, \mu_{I}-1$ lead to a new packet arriving at the head of the SU's buffer queue during a PU OFF period. Therefore, the probability of a packet arriving at the head of the SU's buffer queue during a PU OFF period is given by

$$
\begin{aligned}
\gamma_{I} & =\frac{\mu_{I}-1}{\mu_{I}} \\
& =1-p_{I} .
\end{aligned}
$$

Thus, the CF of the service time for the second scenario is given by

$$
\begin{aligned}
\Phi_{S}(\omega)= & \gamma_{I} \sum_{r=1}^{+\infty} \operatorname{Pr}\left\{I_{\text {periods }}=r\right\} \cdot \Phi_{L}(\omega) \cdot \Phi_{B}^{r-1}(\omega) \\
& +\left(1-\gamma_{I}\right) \sum_{r=1}^{+\infty} \operatorname{Pr}\left\{I_{\text {periods }}=r\right\} \cdot \Phi_{L}(\omega) \cdot \Phi_{B}^{r}(\omega) .
\end{aligned}
$$

Lemma 3: The CF of the packet service time for a general scenario, i.e., without any restrictions regarding the beginning of the packet's transmission and the SU's traffic condition (saturated or non-saturated), is given by

$$
\begin{aligned}
\Phi_{S}(\omega) & \\
= & \left(1-p_{I}+p_{Q E}\left(-1+p_{I}+P_{P U}^{I}\right)\right) \sum_{r=1}^{+\infty} \operatorname{Pr}\left\{I_{\text {periods }}=r\right\} \\
& \times \frac{p_{L} e^{j \omega}}{1-\left(1-p_{L}\right) e^{j \omega}}\left(\frac{p_{B} e^{j \omega}}{1-\left(1-p_{B}\right) e^{j \omega}}\right)^{r-1} \\
& +\left(p_{I}-p_{Q E}\left(-1+p_{I}+P_{P U}^{I}\right)\right) \sum_{r=1}^{+\infty} \operatorname{Pr}\left\{I_{\text {periods }}=r\right\} \\
& \times \frac{p_{L} e^{j \omega}}{1-\left(1-p_{L}\right) e^{j \omega}}\left(\frac{p_{B} e^{j \omega}}{1-\left(1-p_{B}\right) e^{j \omega}}\right)^{r} .
\end{aligned}
$$

Proof: This lemma considers a more general scenario without restrictions about i) the beginning of the data packet 